\title{
Design and optical characterisation of an efficient light trapping structure for dye-sensitized solar cell integrated windows
}

\author{
Andrew Knott ${ }^{1,2}$, Xiao Liu' ${ }^{2}$, Oleg Makarovskiy' ${ }^{1}$, James O'Shea $^{1}$, Chris Tuck ${ }^{2}$, Yupeng Wu ${ }^{2}(\varangle)$ \\ 1. School of Physics and Astronomy, The University of Nottingham, NG7 2RD, UK \\ 2. Faculty of Engineering, The University of Nottingham, NG7 2RD, UK
}

\begin{abstract}
Windows integrated with semi-transparent photovoltaics (PVs) such as Dye-Sensitized Solar Cells (DSSCs) show good potential in improving building performance, in terms of providing daylight, reducing unnecessary solar heat gain and also generating electricity onsite. However, low cell efficiency remains an obstacle for their applications in windows. Using light trapping structures in DSSCs shows the potential to improve solar to electrical conversion efficiency. In this work, different pyramid-patterned titanium dioxide $\left(\mathrm{TiO}_{2}\right)$ geometries are designed to enhance the photon absorption in DSSCs, and characterised using a Monte-Carlo algorithm based 3D ray-tracing simulation. Various studies were carried out under average irradiation, spectrum dependent irradiation and different solar incidental angles, respectively. The simulation results at the average irradiation wavelength $(540 \mathrm{~nm}$ ) were compared to those from a previous study using Scanning Photocurrent Microscopy (SPCM) and a reasonable agreement has been achieved. It was found that the structure based on the pyramid array of side wall angle $54.7^{\circ}$ can significantly enhance light absorption by up to $\sim 25 \%$ and the maximum achievable photocurrent density (MAPD) by up to $\sim 45 \%$ across the spectrum of $380-800 \mathrm{~nm}$, when compared to a planar control counterpart.
\end{abstract}

\author{
Keywords \\ light trapping, \\ ray-tracing, \\ pyramid, \\ pattern, \\ MAPD, \\ wavelength \\ Article History \\ Received: 28 June 2018 \\ Revised: 12 August 2018 \\ Accepted: 30 September 2018 \\ (c) The Author(s) 2018. This article \\ is published with open access at \\ link.Springer.com
}

\section{Introduction}

The last couple of decades have observed a rapid development of advanced window technologies in improving buildings' performance. As a promising multifunctional window, Photovoltaics (PV) integrated windows can directly harvest irradiation to produce electricity on site, but also protect the interiors from overheating and discomfort glare. Currently, traditional silicon-based PV windows are dominating in the market (Skandalos and Karamanis 2015), but a range of thin film solar cells such as Dye-Sensitized Solar Cells (DSSCs) (Grätzel 2003; Kang et al. 2003; Takeda et al. 2009; Lee et al. 2014; Lee and Yoon 2018; Bouvard et al. 2015), polymer solar cells (Chen et al. 2012), Perovskite-based solar cells (Cannavale et al. 2017) and compound semiconductors like cadmium telluride (CdTe) (Casini 2016) have emerged and revolutionized the field of semi-transparent building integrated PVs (BIPVs). Among these, DSSC is regarded as one of the most promising alternatives to silicon solar cells, because of its low cost, simple fabrication, tuned transparency and workability under low-light conditions (Fakharuddin et al. 2014; Gong et al. 2017). Nevertheless, DSSCs are still under research and development stage, and further efforts are required to improve power conversion efficiency (PCE) and overcome issues such as chemical degradation and liquid electrolyte leakage (Cannavale et al. 2017; Gong et al. 2017).

DSSC is a photoelectrochemical PV device in which light harvesting occurs at the photoanode that consists of a transparent conductive oxide (TCO) electrode and a mesoporous active layer (typically titanium dioxide $\left(\mathrm{TiO}_{2}\right)$ ) with dyes absorbed on it (Gagliardi and Falconieri 2015; Gong et al. 2017). Using a thicker active layer seems to be a simple approach to enhance light absorption thus leading to a higher PCE; however, the intrinsic defects associated with charge-carrier diffusion path length and recombination loss often limits the layer thickness (Cho et al. 2013; Foster and John 2013; Wooh et al. 2013). In recent years, numerous researches have been made in developing light trapping

E-mail: Yupeng.Wu@nottingham.ac.uk 
structures to increase the multiple reflections and absorption path lengths inside photoanodes to enhance DSSC conversion efficiencies. In the study of Wang et al. (2012), DSSCs were fabricated on fluorine doped tin oxide (FTO) glass substrates where patterned periodic arrays of nanopillars and nanolines were generated. The incident photon-to-current conversion efficiencies (IPCEs) of the nanopatterned structures in the $400-650 \mathrm{~nm}$ wavelength range were measured to be $2 \%-5 \%$ higher than that without nanopatterns. Kim et al. (2012) prepared a N719-dye-coated $\mathrm{TiO}_{2}$ film with $11 \mu \mathrm{m}$ thickness and $250 \mathrm{~nm}$ wide periodic nanoline patterns, and revealed that the nanopatterning enables stronger back-reflection for light trapping in the active layer compared to a simple planar structure. Foster and John (2013) proposed a DSSC consisting of a periodic array of $\mathrm{TiO}_{2}$ nanotubes filled with C101-dye-coated $\mathrm{TiO}_{2}$ nanoparticles. The nanotube diameter and spacing were optimised to concentrate light within the naotubes interior. The researchers adopted a coupled ray-tracing and electrical model (published by Wenger et al. (2011)) for 2D simulations, and found the design improves the maximum achievable photocurrent density (MAPD) by $33 \%$ relative to a planar counterpart. Wooh et al. (2013) compared DSSCs with different 3D micro-patterned $\mathrm{TiO}_{2}$ structures, including pillar, prism, pyramid and inversepyramid patterned geometries. Among these, the pyramidpatterned $\mathrm{TiO}_{2}$ structure was observed to have the highest UV-vis light absorption and photocurrent density generation. The experimental results reach good agreement with the findings on the optical path lengths in different photoanode geometries, which were calculated by a commercial ray-tracing simulation package LightTools. Yun et al. (2017) developed 3D micro-patterned hexagonal-shaped photoanodes of 100-1000 $\mu \mathrm{m}$ pattern sizes and $30 \mu \mathrm{m}$ pattern spacing, and demonstrated that a high DSSC conversion efficiency can be achieved by controlling the photoanode thickness and pattern size. Zhang et al. (2015) measured the IPCE spectra of the DSSCs fabricated on grating-grid and orthogonalgrid patterned $\mathrm{TiO}_{2}$ films in the wavelength range of $400-750 \mathrm{~nm}$. It was found that the IPCEs increase over the whole spectrum as the pattern grid spacings drop from 40 to $5 \mu \mathrm{m}$. Moreover, a wavelength-selectivity of light trapping was observed when changing the grid spacings, which may be attributed to the mismatch between the spectrum of light scattered by the patterns and the dye absorption spectrum. Recently, Knott et al. (2018) utilized a submicron 3D-pinting technique known as Two-Photon Polymerization (TPP) to develop light trapping structures for DSSCs. In the study, periodic pyramid-patterned $\mathrm{TiO}_{2}$ microstructures were prepared and tested by the Scanning Photocurrent Microscopy (SPCM) experiments and showed significant enhancement effect of up to $\sim 25 \%$ on photocurrent in the cell when compared to a non-patterned planar structure.
Until now, there have already been many attempts to fabricate DSSCs based on periodic micro or nano-patterned photoanode geometries and to characterize their light trapping behaviours by experiments. However, to our knowledge, only a little literature specializes in optically modelling the light trapping in 3D periodic patterned structures and linking the DSSCs' optical-electrical characteristics with the variation of geometrical parameters and incident light conditions. In this study, we adopt a 3D ray-tracing technique based on Monte-Carlo algorithm to investigate the light trapping effectiveness of the designed DSSCs based on periodic pyramid-patterned $\mathrm{TiO}_{2}$ films. The DSSC performance at a single wavelength $(540 \mathrm{~nm})$ is simulated and compared to the SPCM results obtained in the previous work (Knott et al. 2018). The DSSC performance at different incident light angles, over AM1.5 solar spectrum and with different pyramid geometries are also evaluated.

\section{Methodology}

$3 \mathrm{D}$ ray-tracing models are developed for our light trapping designs which have been incorporated into a DSSC device (Knott et al. 2018). The device consists of a glass substrate with a transparent conducting FTO coating, $\mathrm{TiO}_{2}$ active layer, electrolyte and finally another FTO coated conducting glass back contact. The glass substrate is $3 \mathrm{~mm}$ (Pilkington, TEC15) the thickness of which has been found by previous work (Foster and John 2013) to not affect absorption in the active layer and as such is set to $1 \mu \mathrm{m}$ in the model to save computational time. The FTO coating thickness is $500 \mathrm{~nm}$. The active layer of dyed $\mathrm{TiO}_{2}$ is $10 \mu \mathrm{m}$ thick (i.e. $5 \mu \mathrm{m}$ pyramid structure on $5 \mu \mathrm{m}$ base). The areas in-between structures are considered to be pure electrolyte. The electrolyte layer is also set to $1 \mu \mathrm{m}$ in the interests of saving computational time (Foster and John 2013). All of these layers are in contact i.e. no air gap between. Although in reality the glass substrate, FTO and electrolyte all absorb some light, for the purposes of this study we ignore these effects in all the simulations as we are only focusing on the enhancement of absorption in the active layer (Dabirian and Taghavinia 2015). A schematic diagram of the DSSC model can be seen in Fig. 1(a). The patterned array for side wall angle of $54.7^{\circ}$ is $90 \mu \mathrm{m} \times 90 \mu \mathrm{m}$ with $10 \times 10$ pyramids. The pyramid geometry and dimensions can be seen in Fig. 1(b). The structures have a $1 \mu \mathrm{m}$ plateau at the pyramid apex and a $1 \mu \mathrm{m}$ gap between pyramids in the array. The designed and developed DSSC windows are displayed in Figs. 1(c) and (d).

In the control sample the thickness is calculated to reflect the same volume as the pyramid array, when this volume is redistributed into a planar geometry the thickness is $8.5 \mu \mathrm{m}$ for pyramid angle side walls of $54.7^{\circ}$ and height $5 \mu \mathrm{m}$. This ensures that absorption enhancement is due to light trapping 
(a)

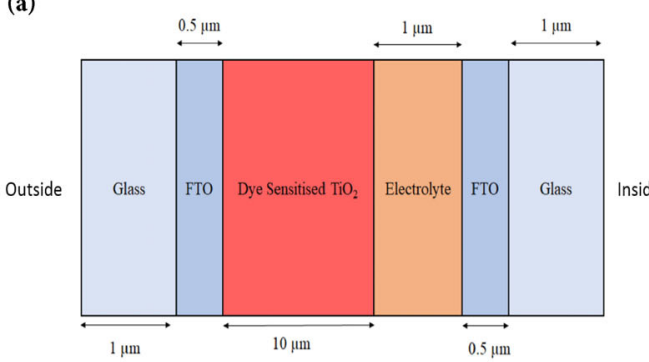

(c)

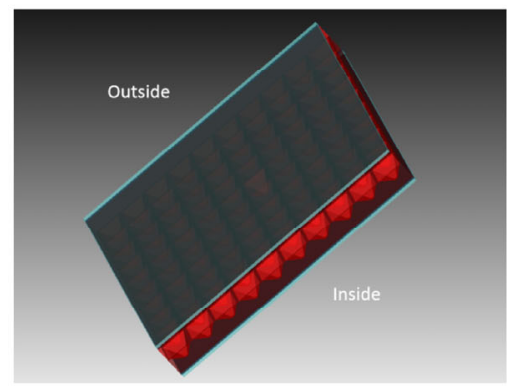

(b)

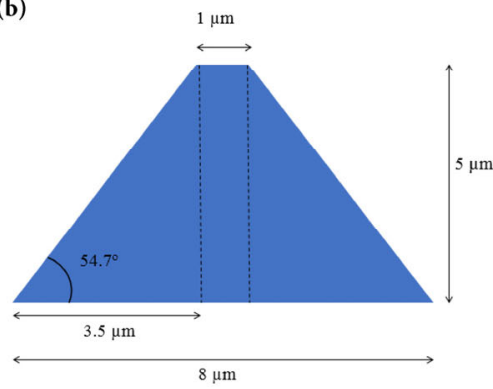

(d)

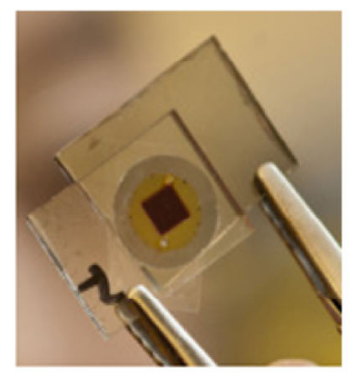

Fig. 1 (a) Schematic of DSSC device stack used in model, (b) geometry of pyramid with side wall angels of $54.7^{\circ},(c)$ proposed $\mathrm{TiO}_{2}$ light trapping periodic pyramid array, and (d) developed DSSC system with the patterned $\mathrm{TiO}_{2}$ layer

rather than differences in the amount of active material. This approach is also taken when looking at pyramid arrays of differing geometries.

Materials properties are all obtained from experimental data in the literature, with the values for the refractive index of the glass, FTO and $\mathrm{TiO}_{2}$ provide by Ball et al. (2015), the values for the refractive index of the electrolyte solvent by Kozma et al. (2005) and Moutzouris et al. (2014). Absorption data for $\mathrm{TiO}_{2}$ dyed with N719 (Ruthenizer 535-bisTBA, Solaronix) is provided by Ito et al. (2013). Incident rays with intensity of $1000 \mathrm{~W} / \mathrm{m}^{2}$ at the average wavelength of solar radiation $(540 \mathrm{~nm})$ are used for single wavelength studies. All light is incident at $0^{\circ}$ except in angle dependence tests. Table 1 shows the properties of the materials used in the model at 540nm. Further simulations look the absorption over the AM 1.5 solar spectrum from 380 to $800 \mathrm{~nm}$. This is the region of high absorption for the dye N719 and the range with absorption data available in the literature. Next a number of variations on the structure used experimentally are studied, alongside further pyramid structures of differing side wall angle. For these studies the height of all the pyramids is kept constant at $5 \mu \mathrm{m}$.

Table 1 Refractive indices and absorption coefficients of the DSSC components

\begin{tabular}{lcc}
\hline \multicolumn{1}{c}{ Material } & Refractive index, $n$ & Absorption coefficient, $\alpha$ \\
\hline $\mathrm{TiO}_{2}+\mathrm{N} 719$ & 2.13 & $0.11 \mu \mathrm{m}^{-1}$ \\
$\mathrm{FTO}$ & 1.90 & $0 \mu \mathrm{m}^{-1}$ \\
Glass & 1.51 & $0 \mu \mathrm{m}^{-1}$ \\
Electrolyte & 1.34 & $0 \mu \mathrm{m}^{-1}$ \\
\hline
\end{tabular}

For this model ray simulations are conducted using the commercial raytracing software Tracepro (Lambda Research Corporation), where rays that enter a material with no zero absorption transmit light according to the Beer-Lambert law (see Eq. (1)).

$\Phi_{\mathrm{T}}=\Phi_{0} \mathrm{e}^{-\alpha t}$

where $\Phi_{\mathrm{T}}$ and $\Phi_{0}$ are the transmitted and incidence flux, respectively, $\alpha$ is the absorption coefficient and $t$ is the material thickness through which the ray has travelled.

The flux absorbed by the material is then given by Eq. (2).

$\Phi_{\mathrm{A}}=\Phi_{0}\left(1-\mathrm{e}^{-\alpha t}\right)$

From this, absorption in our DSSC light trapping devices can be simulated. Then the spectral photocurrent density and the maximum achievable photocurrent density (MAPD) can be calculated for over the AM 1.5 spectrum. The spectral photocurrent density is given by Eq. (3).

$J(\lambda, \theta)=\frac{e \lambda}{h c} I(\lambda) A(\lambda, \theta)$

And the maximum achievable photocurrent density (MAPD) is given by Eq. (4).

$J_{\lambda_{\min }}^{\lambda_{\max }}(\theta)=\int_{\lambda_{\min }}^{\lambda_{\max }} \frac{e \lambda}{h c} I(\lambda) A(\lambda, \theta) \mathrm{d} \lambda$

where $e$ is the electron charge, $h$ is Planck's constant, and $c$ is the speed of light in a vacuum. $I(\lambda)$ is the AM 1.5 solar light intensity incident on the cell per unit wavelength of 
incident angle $\theta$. $A$ is the normalised absorption of the device, the relation $(\lambda) A(\lambda, \theta)$ is divided by the photon energy $h c / \lambda$ to give the number of photons absorbed per second per unit area per unit wavelength. The MAPD assumes perfect charge collection whereby one absorbed photon results in one electric charge contributing to current (no losses through recombination's) and is obtained by multiplying by $e$ and integrating over relevant wavelengths. Therefore, experimental short circuit current densities are lower than the MAPD.

In the Ray-tracing simulation, Monte-Carlo method is used to characterise the scattering light upon striking a surface. Here a computer algorithm relies on repeated random sampling to obtain result for a deterministic process, when this process is repeated a large number of times and the results are aggregated an accurate picture of the optical model can be simulated. The light ray incident on a given surface is distributed to random rays according to a probability distribution determined the material properties (absorbance, reflectance, transmittance, refractive index), before moving onto to the next surface/interface. The larger the number of incident rays the more accurate the calculation will become; however, it is a trade of between accuracy and computational time. Because of this it is necessary to perform a ray independence test, whereby the total number of rays is increased until the errors in the result of the simulation become insignificant.

Ray-independence test was conducted at $540 \mathrm{~nm}$ for both the control and the pyramid patterned array. Fig. 2 shows the ray independence test for the pyramid array at $540 \mathrm{~nm}$, with errors becoming negligible from 100,000 rays. Since simulations using $1,000,000$ rays did not result in excessive computation times this number was selected for further studies to ensure any computational errors were completely removed. The errors for the control become negligible at only 10,000 rays however further simulations were also conducted at 1,000,000 for consistency.

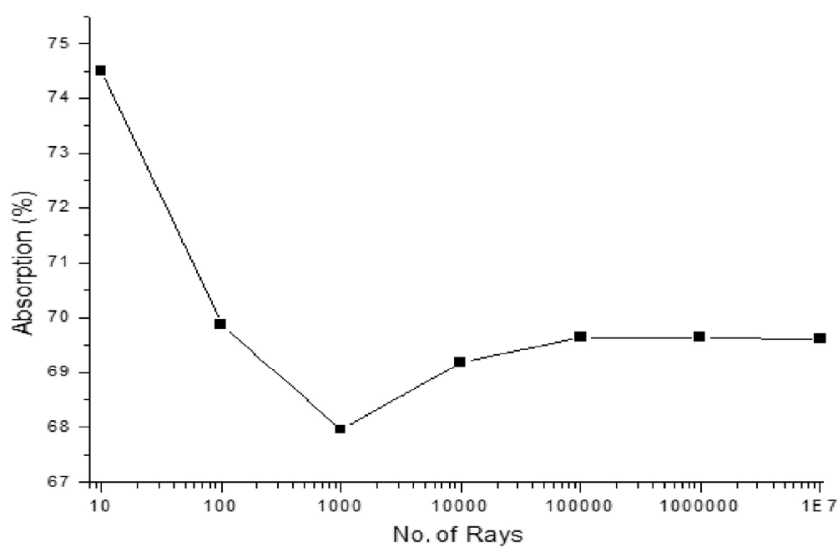

Fig. 2 Ray-independence tests for pyramid array patterned $\mathrm{TiO}_{2}$ electrode at $540 \mathrm{~nm}$

\section{Results}

Ray tracing simulation results will begin with single wavelength studies at the average of the solar spectrum $(540 \mathrm{~nm})$, incidentally this is also close to a peak in absorption for the dye N719 which is used in these simulations and all light trapping experimental results to follow. Our light trapping structures will be compared against a planar control device. The absorption's dependence on the incidence angle of the light will next be presented (at $540 \mathrm{~nm}$ ) before the structures are compared across the solar AM1.5 spectrum.

Further results to follow will look at the variations in the light trapping structures made to help facilitate their microfabrication whilst maintaining high structure quality across the array and how these changes can affect absorption enhancement. Finally results from a number of pyramid arrays of differing geometries will be shown.

\subsection{Pyramid array of side wall angle $54.7^{\circ}$}

The ray-tracing simulation results begin with a model of our light trapping designs which were used for the fabrication of patterned electrodes (Knott et al. 2018). These structures have a side wall angle of $54.7^{\circ}$, height $5 \mu \mathrm{m}$ and base diameter $8 \mu \mathrm{m}$. These structures also have a $1 \mu \mathrm{m}$ plateau at the pyramid apex and a $1 \mu \mathrm{m}$ gap between pyramids in the array, with these modifications to the design being made for practical reasons to ensure high quality structures during microfabrication. Structure geometry can be seen in Fig. 1(b).

\subsubsection{Single wavelength}

The first simulation results are at single wavelength of the average solar spectrum (540 $\mathrm{nm}$ ) as previously mentioned. Under these conditions the control model was found to be absorb $68 \%$ of incident light, compared to our light trapping structures which absorbed $86 \%$. An increase of total light absorbed by $18 \%$ or an enhancement in absorption by $\sim 30 \%$ over the planar control. The increased absorption is attributed to the increased path length of light propagating through the active layer. This can be seen in a visual representation in Fig. 3 where only one ray is traced in the simulation for both our light trapping structures and the planar control. Note that the pyramids and base in Fig. 3(a) are both the same material but the pyramids are represented in a different colour due to the electrolyte which is in-between the pyramids.

Although absorption and photocurrent generation do not perfectly correlate, absorption is the most important contributing mechanism for this process. We can expect experimental results of photocurrent enhancement to be in the same region as absorption enhancements, although somewhat less due to recombination losses. SPCM results 
(a)

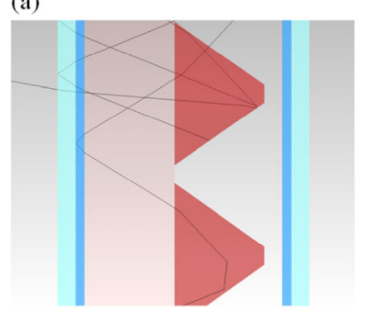

(b)

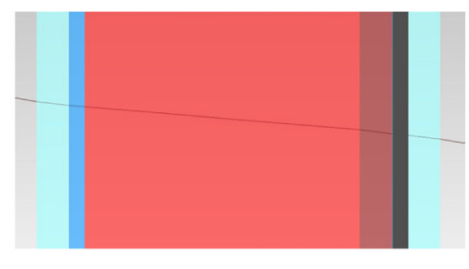

Fig. 3 Visual aid showing how light can propagate through DSSC device for with (a) pyramid array and (b) planar device, at $10^{\circ}$ incident angle. Note increased path length for pyramid array

were conducted under laser irradiation at $405 \mathrm{~nm}$. Simulation results at this wavelength show $16 \%$ more total light absorbed, or an enhancement in absorption by $\sim 22 \%$. Experimental results (Knott et al. 2018) show enhancement in photocurrent generation up to maximum of $\sim 25 \%$ although mean enhancement is lower at $\sim 10 \%$. Both results show reasonable agreement, with experimental work showing lower photocurrent enhancement than the absorption enhancement in the simulation as expected, indicating the model is effective.

From the simulations, it is found that the transmittances of the pyramid-patterned DSSC window and the planar control DSSC window are $\sim 11 \%$ and $\sim 29 \%$ respectively.

\subsubsection{Incident angle dependence}

The absorptions dependence on the incident angle of the light is simulated from $0^{\circ}$ to $85^{\circ}$ for both the control and the light trapping structures at a wavelength of $540 \mathrm{~nm}$. Results can be seen in Fig. 4. As previously mentioned the light trapping structures absorb $18 \%$ more of total incident light at $0^{\circ}$. In both systems absorption remains stable with only minor fluctuations up to $40^{\circ}$. Here absorption starts to decline for both systems although interestingly more dramatically for our light trapping designs, closing the gap in absorption

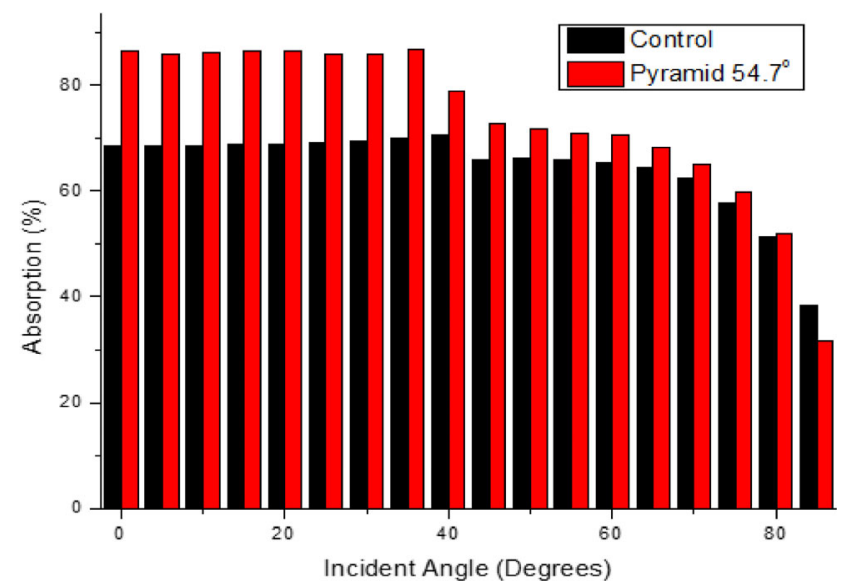

Fig. 4 Dependence of absorption on incidence angle of light of wavelength $540 \mathrm{~nm}$ for planar control and pyramids of side wall angle $54.7^{\circ}$ between the two. Despite this our light trapping designs outperform the planar control at all angles up until $85^{\circ}$. At this final angle absorption in the planar control is $38 \%$ compared to $32 \%$ for our light trapping designs. Overall, we see a drop in total absorption from $0^{\circ}$ to $85^{\circ}$ of $30 \%$ for the planar control and $55 \%$ for the light trapping structures. Despite the larger drop in absorption for the light trapping structures they still outperform the planar control overall.

\subsubsection{Spectral dependence and MAPD}

Next, simulations were conducted for the same structures (control and light trapping) over the AM1.5 solar spectrum from 380 to $800 \mathrm{~nm}$. Results can be seen in Fig. 5(a), which shows that the light trapping structures absorb a higher percentage of light across this entire wavelength range. There is a dip in absorption for both systems at $450 \mathrm{~nm}$. However, interestingly this is less dramatic for our light trapping structures than the planar control. With the difference between the first maxima and the first minima for the control being $15 \%$ compared to only $8 \%$ for our light trapping results. Fig. 5(b) shows the increase in absorption for our structures and the planar control across this spectrum, where there is significant variation ranging from $\sim 15 \%$ to $\sim 25 \%$ with the greatest enhancement seen between 550 and $700 \mathrm{~nm}$. It is also worth mentioning the performance of both systems at $405 \mathrm{~nm}$ since it is this wavelength which is used for illumination in our SPCM experiments. Here the control sample absorbs $71 \%$ of incident light and the light trapping structures absorb $87 \%$, an increase of $16 \%$ and close to the maximum absorption for both systems.

Fig. 6(a) shows spectral photocurrent density plotted against wavelength over the AM1.5 solar spectrum from 380 to $800 \mathrm{~nm}$ assuming an internal quantum efficiency of 100\%. And Fig. 6(b) shows the photocurrent enhancement of the light trapping device over the planar control. This gives us more information about how the devices will perform under their intended conditions of solar irradiation. Although the devices reach their maximum absorption close to $380 \mathrm{~nm}$ the low power of solar irradiation at this wavelength means we see the lowest value of photocurrent density. The maximum achievable photocurrent density (MAPD) is calculated to be $11.22 \mathrm{~mA} / \mathrm{cm}^{2}$ for the control and $16.55 \mathrm{~mA} / \mathrm{cm}^{2}$ for our light trapping device, an improvement of $\sim 45 \%$. MAPD values agree well with experimental reports of the short circuit current obtained for DSSC using the dye N719 (Ito et al. 2008, 2013), helping to further validate the model. These results cannot be directly compared with experimental SPCM results as they were conducted under laser irradiation of wavelength $405 \mathrm{~nm}$ and recombination losses are not taken into account. But they are a good indication that we should see significant enhancement of the photocurrent generation in these experiments. 
(a)

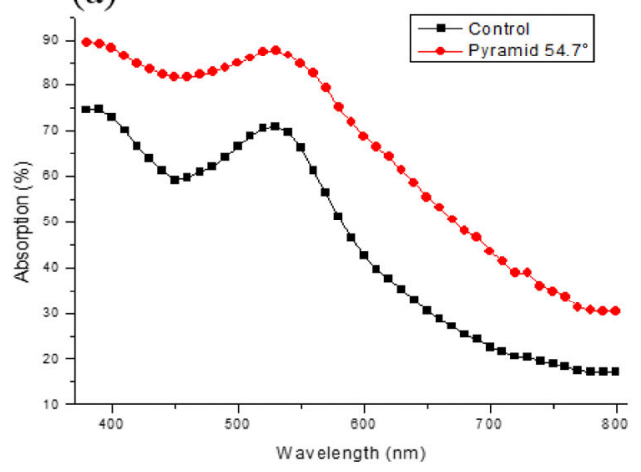

(b)

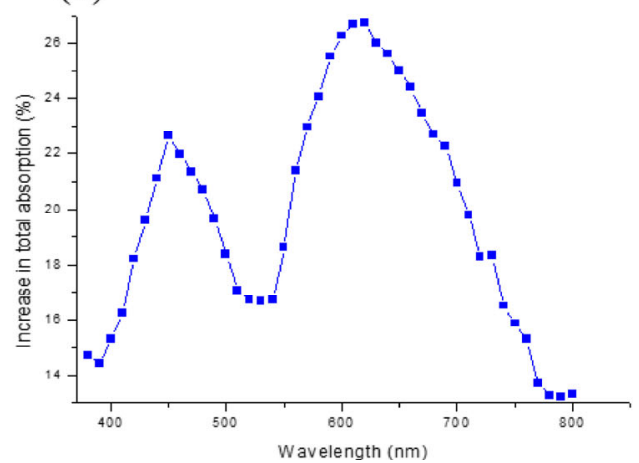

Fig. 5 (a) Absorption over spectrum from 380 to $800 \mathrm{~nm}$ for planar control and pyramid array of side wall angle $54.7^{\circ}$. (b) Increase in total absorption in light trapping DSSC

(a)

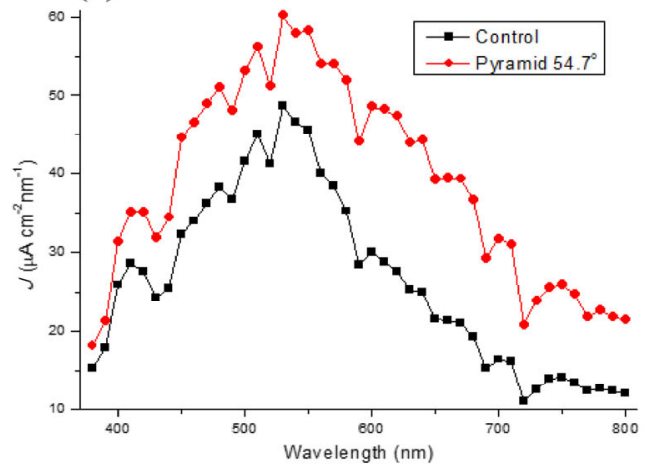

(b)

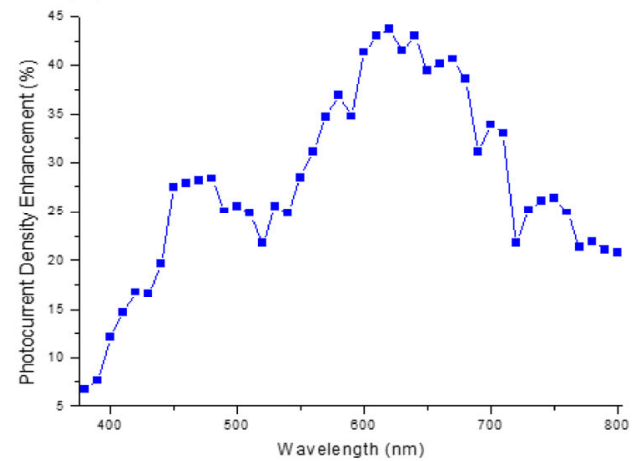

Fig. 6 (a) Simulated spectral current-density plotted as a function of wavelength over the AM1.5 solar spectrum from 380 to $800 \mathrm{~nm}$. (b) Spectral photocurrent enhancement of light trapping model over planar control

\subsection{Variation of pyramid array of side wall angle $54.7^{\circ}$}

With adaptations to our designs made to help facilitate the high-quality microfabrication of the pyramids arrays. The next set of simulations were conducted to look at the effects these changes have on absorption. Fig. 7 shows the four different pyramid structures compared in these results. The structures fabricated in the experimental work and studied in the previous simulations detailed above can be seen in Fig. 7(a), containing both modifications of the plateau and the gap between pyramids in the array and named 'Pyramid $54.7^{\circ}$. Next, the so named 'Perfect Pyramid' can be seen in Fig. 7(b), containing no plateau and no gaps. Followed by the structure where there is a gap between pyramids in the array but no plateau seen in Fig. 7(c), named 'Gaps Only'. Finally, the structures with a plateau at the pyramid apex but no gaps between pyramids in the array named as 'Plateau Only'.

Results for these four structures and the planar control are shown in Fig. 8. Here, it can be seen that when these modifications are removed the structures perform better, with the 'Perfect Pyramid' structure absorbing up to $10 \%$ more light then the structures used experimentally. The
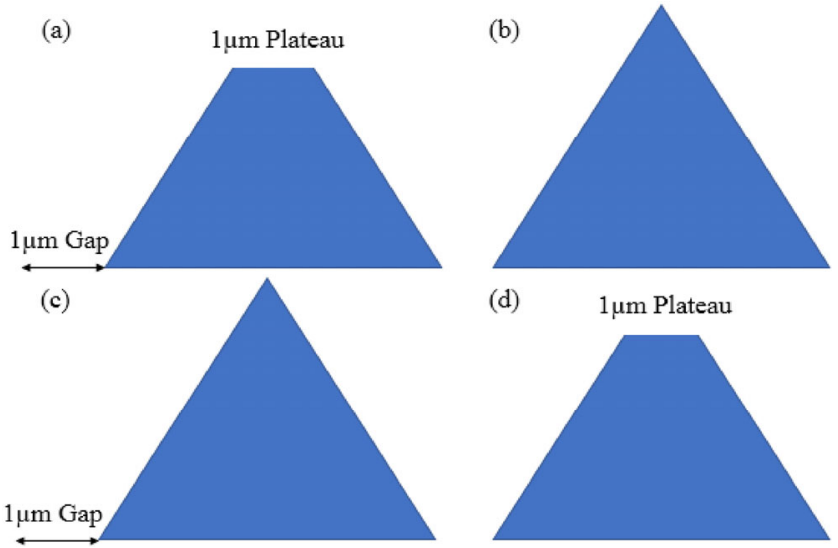

(d)

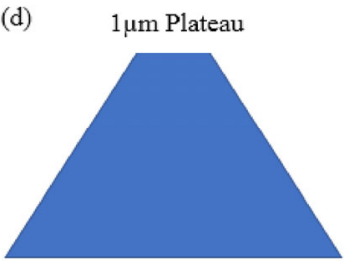

Fig. 7 Different variations of pyramid of side wall angle $54.7^{\circ}$, where adaptations were made for practical reasons during microfabrication. (a) Pyramid geometry fabricated experimentally with $1 \mathrm{~m}$ gap between pyramids and $1 \mathrm{~m}$ plateau at pyramid apex. (b) Perfect pyramid array with no gaps and no plateau. (c) Gaps between pyramids only. (d) Plateau at pyramid apex only

structures where only one modification is removed also absorb more light than the 'Pyramid 54.7'; however not as much as the 'Perfect Pyramid'. This is to be expected as for each modification there is a greater surface area of the 
(a)

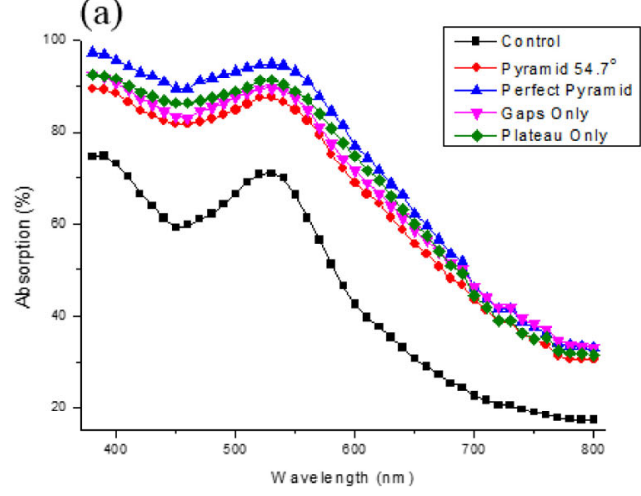

(b)

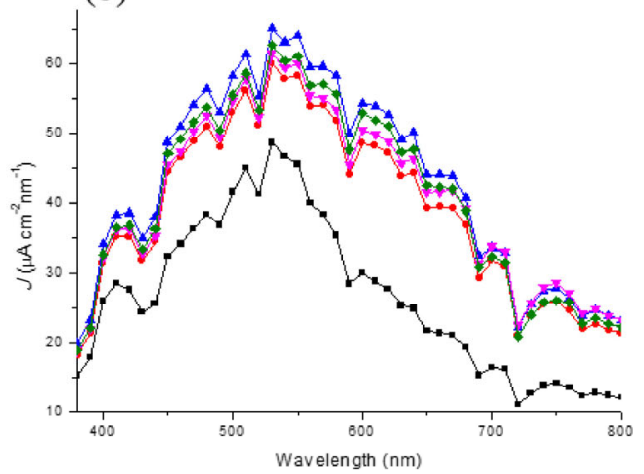

Fig. 8 (a) Graph showing the effects structure adaptations made during microfabrication have on absorption enhancement in the device. (b) Spectral photocurrent density for pyramid modifications

array which is planar, increasing the chance that light will be not reflected back into the active layer, thus reducing the overall path length of the light in the active layer leading to a drop in absorption. Surprisingly this difference reduces at longer wavelengths where the difference in all four structures falls to only a few percent. Fig. 8(b) shows the corresponding spectral photocurrent density for these structure modifications where the enhancements in absorption are reflected, their calculated MAPD and its comparison with all other simulated devices can be seen in Table 2 .

\subsection{Pyramid arrays of differing geometries}

Finally, simulations were repeated for differing pyramid geometries. Here the height is kept constant and side wall angle is changed with angles of $30^{\circ}, 40^{\circ}, 60^{\circ}$ and $80^{\circ}$ being studied. Note that the modifications made for fabrication are not implemented here, and all are considered to be 'Perfect Pyramids' with no gaps and no plateau at the pyramid apex.

Results are shown in Fig. 9 where is can be seen that absorption is higher for only pyramids of side wall angle $40^{\circ}$ than those physically fabricated and characterised, however this is only by a small amount. And at larger wavelengths,

Table 2 Calculated MAPD for all device geometries simulated

\begin{tabular}{lc}
\hline \multicolumn{1}{c}{ DSSC geometry } & MAPD $\left(\mathrm{mA} / \mathrm{cm}^{2}\right)$ \\
\hline Planar geometry & 11.22 \\
Pyramid 54.7' & 16.55 \\
'Perfect Pyramid' & 18.21 \\
'Gaps Only' & 17.25 \\
'Plateau Only' & 17.42 \\
$30^{\circ}$ & 10.49 \\
$40^{\circ}$ & 17.71 \\
$60^{\circ}$ & 17.56 \\
$80^{\circ}$ & 13.81 \\
\hline
\end{tabular}

where there is less absorption, those pyramids of side wall angle $54.7^{\circ}$ perform better than even these. All pyramid arrays perform better than the planar control apart from pyramids of side wall angles $30^{\circ}$ which surprisingly performs worse at shorter wavelengths and matches the planar absorption at longer wavelengths.

At longer wavelengths $(>650 \mathrm{~nm})$ there is much more variation in the percentage of incident light absorbed across the different geometries. Here our structures fabricated and characterised experimentally outperform all other pyramid geometries apart from those of side wall angles of $60^{\circ}$ which absorb a few percent more at wavelengths greater than $740 \mathrm{~nm}$.

Generally, it can be said that all structures with a side wall angle of greater than $30^{\circ}$ absorb more light than the planar control device. With the maximum enhancement seen in structures with side walls angle of $40^{\circ}, 54.7^{\circ}$ and $60^{\circ}$ which are only separated by a few percent up to $650 \mathrm{~nm}$ after which the variation increases. Pyramids with the steepest side wall angle of $80^{\circ}$ still outperform the planar control but are significantly worse than the three best performing geometries. The corresponding spectral photocurrent density can be seen in Fig. 9(b) where the enhancements in absorption are reflected, the calculated MAPD can be seen in Table 2 where the best light trapping structures show impressive improvements over the planar control.

\section{Conclusions}

In this work, 3D ray-tracing simulations based on MonteCarlo method are performed in aid of the design and development of micropatterned 3D pyramidal $\mathrm{TiO}_{2}$ films in DSSCs to enhance light trapping performance. The designed pyramid structure with side wall angle of $54.7^{\circ}$ is firstly tested at an average irradiation wavelength $(540 \mathrm{~nm})$ and a reasonable agreement is achieved between the ray-tracing simulation results and the SPCM experiment results. Afterwards, the 

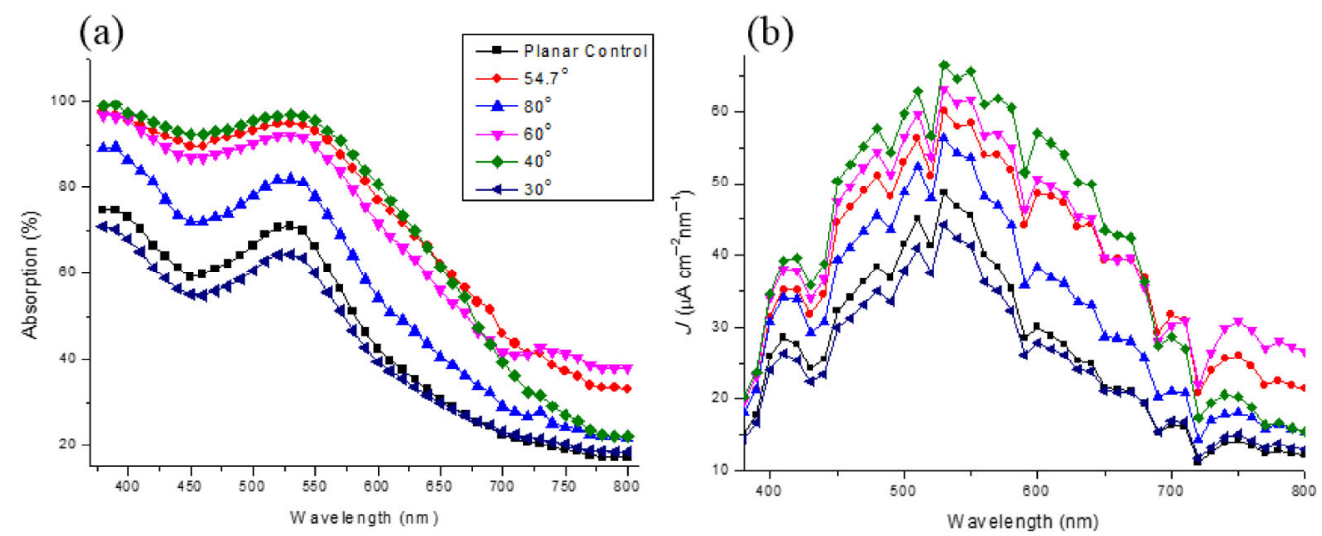

Fig. 9 (a) Graph showing absorption for a number of pyramid arrays with differing side wall angles. (b) Spectral photocurrent density for pyramid modification

same geometry is simulated at different incident angles over the AM1.5 spectrum (from 380 to $800 \mathrm{~nm}$ ). Significant improvement of light absorption (between $\sim 15$ and $~ 25 \%$ ) and MAPD ( $45 \%$ at maximum) are observed when compared to the planar control counterpart. Simulations are also carried out for different pyramid structures in terms of pattern shapes (with or without plateau and gap) and side wall angles $\left(30^{\circ}, 40^{\circ}, 60^{\circ}\right.$ and $\left.80^{\circ}\right)$ with corresponding MAPDs calculated. Future work will focus on extending the designed DSSC geometries to window integration to improve buildings' daylighting control and energy saving and generation performance.

\section{Acknowledgements}

This work was supported by the School of Physics and Astronomy and Faculty of Engineering, University of Nottingham through a joint $\mathrm{PhD}$ studentship awarded to Andrew Knott.

Open Access: This article is distributed under the terms of the Creative Commons Attribution 4.0 International License (http://creativecommons.org/licenses/by/4.0/), which permits unrestricted use, distribution, and reproduction in any medium, provided you give appropriate credit to the original author(s) and the source, provide a link to the Creative Commons license, and indicate if changes were made.

\section{References}

Ball JM, Stranks SD, Hörantner MT, Hüttner S, Zhang W, Crossland EJW, Ramirez I, Riede M, Hohnston MB, Friend RH, Snaith HJ (2015). Optical properties and limiting photocurrent of thin-film perovskite solar cells. Energy \& Environmental Science, 8: 602-609.

Bouvard O, Vanzo S, Schüler A (2015). Experimental determination of optical and thermal properties of semi-transparent photovoltaic modules based on dye-sensitized solar cells. Energy Procedia, 78: 453-458.
Cannavale A, Hörantner M, Eperon GE, Snaith HJ, Fiorito f, Ayr U, Martellotta F (2017). Building integration of semitransparent perovskite-based solar cells: Energy performance and visual comfort assessment. Applied Energy, 194: 94-107.

Casini M (2016). Smart Buildings: Advanced Materials and Nanotechnology to Improve Energy-Efficiency and Environmental Performance. Duxford UK: Woodhead Publishing.

Chen K-S, Salinas J-F, Yip H-L, Huo L, Hou J, Jen AK-Y (2012). Semi-transparent polymer solar cells with 6\% PCE, 25\% average visible transmittance and a color rendering index close to 100 for power generating window applications. Energy \& Environmental Science, 5: 9551-9557.

Cho C, Kim H, Jeong S, Baek S-W, Seo J-W, Han D, Kim K, Park Y, Yoo S, Lee J-Y (2013). Random and V-groove texturing for efficient light trapping in organic photovoltaic cells. Solar Energy Materials and Solar Cells, 115: 36-41.

Dabirian A, Taghavinia N (2015). Theoretical study of light trapping in nanostructured thin film solar cells using wavelength-scale silver particles. ACS Applied Materials \& Interfaces, 7: 14926-14932.

Fakharuddin A, Jose R, Brown TM, Fabregat-Santiago F, Bisquert J (2014). A perspective on the production of dye-sensitized solar modules. Energy \& Environmental Science, 7: 3952-3981.

Foster S, John S (2013). Light-trapping in dye-sensitized solar cells. Energy \& Environmental Science, 6: 2972-2983.

Gagliardi S, Falconieri M (2015). Experimental determination of the light-trapping-induced absorption enhancement factor in DSSC photoanodes. Beilstein Journal of Nanotechnology, 6: 886-892.

Gong J, Sumathy K, Qiao Q, Zhou Z (2017). Review on dye-sensitized solar cells (DSSCs): Advanced techniques and research trends. Renewable and Sustainable Energy Reviews, 68: 234-246.

Grätzel M (2003). Dye-sensitized solar cells. Journal of Photochemistry and Photobiology C: Photochemistry Reviews, 4: 145-153.

Ito S, Murakami TN, Comte P, Liska P, Grätzel C, Nazeeruddin MK, Grätzel M (2008). Fabrication of thin film dye sensitized solar cells with solar to electric power conversion efficiency over $10 \%$. Thin Solid Films, 516: 4613-4619.

Ito S, Takahashi K, Yusa S-I, Saito M, Shigetomi T (2013). Ultradurable dye-sensitized solar cells under $120^{\circ} \mathrm{C}$ using cross-linkage dye and ionic-liquid electrolyte. International Journal of Photoenergy, 2013: 501868. 
Kang MG, Park N-G, Park YJ, Ryu KS, Chang SH (2003). Manufacturing method for transparent electric windows using dye-sensitized $\mathrm{TiO}_{2}$ solar cells. Solar Energy Materials and Solar Cells, 75: 475-479.

Kim J, Koh JK, Kim B, Kim JH, Kim E (2012). Nanopatterning of mesoporous inorganic oxide films for efficient light harvesting of dye-sensitized solar cells. Angewandte Chemie, 124: 6970-6975.

Knott A, Makarovskiy O, O'Shea J, Wu Y, Tuck C (2018). Scanning photocurrent microscopy of 3D printed light trapping structures in dye-sensitized solar cells. Solar Energy Materials and Solar Cells, 180: 103-109.

Kozma IZ, Krok P, Riedle E (2005). Direct measurement of the group-velocity mismatch and derivation of the refractive-index dispersion for a variety of solvents in the ultraviolet. Journal of the Optical Society of America B, 22: 1479-1485.

Lee HM, Yoon JH (2018). Power performance analysis of a transparent DSSC BIPV window based on 2 year measurement data in a full-scale mock-up. Applied Energy, 225: 1013-1021.

Lee JW, Park J, Jung H-J (2014). A feasibility study on a building's window system based on dye-sensitized solar cells. Energy and Buildings, 81: 38-47.

Moutzouris K, Papamichael M, Betsis SC, Stavrakas I, Hloupis G, Triantis D (2014). Refractive, dispersive and thermo-optic properties of twelve organic solvents in the visible and near-infrared. Applied Physics B, 116: 617-622.

Skandalos N, Karamanis D (2015). PV glazing technologies. Renewable and Sustainable Energy Reviews, 49: 306-322.
Takeda Y, Kato N, Higuchi K, Takeichi A, Motohiro T, Fukumoto S, Sano T, Toyoda T (2009). Monolithically series-interconnected transparent modules of dye-sensitized solar cells. Solar Energy Materials and Solar Cells, 93: 808-811.

Wang F, Subbaiyan NK, Wang Q, Rochford C, Xu G, Lu R, Elliot A, D'Souza F, Hui R, Wu J (2012). Development of nanopatterned fluorine-doped tin oxide electrodes for dye-sensitized solar cells with improved light trapping. ACS Applied Materials \& Interfaces, 4: 1565-1572.

Wenger S, Schmid M, Rothenberger G, Gentsch A, Gratzel M, Schumacher JO (2011). Coupled optical and electronic modeling of dye-sensitized solar cells for steady-state parameter extraction. The Journal of Physical Chemistry C, 115: 10218-10229.

Wooh S, Yoon H, Jung JH, Lee YG, Koh JH, Lee B, Kang YS, Char K (2013). Efficient light harvesting with micropatterned 3D pyramidal photoanodes in dye-sensitized solar cells. Advanced Materials, 25: 3111-3116.

Yun MJ, Sim YH, Cha SI, Seo SH, Lee DY (2017). High energy conversion efficiency with 3-D micro-patterned photoanode for enhancement diffusivity and modification of photon distribution in dye-sensitized solar cells. Scientific Reports, 7: 15027.

Zhang X, Liu H, Huang X, Jiang H (2015). One-step femtosecond laser patterning of light-trapping structure on dye-sensitized solar cell photoelectrodes. Journal of Materials Chemistry C, 3: 3336-3341. 